\title{
El reciclaje de vidrio y su impacto en la conservación del medio ambiente.
}

The recycling of glass and its impact on the conservation of the environment.

\author{
Dr. Tatiana Carrasco R. ${ }^{1}$
}

\section{Resumen.}

Este documento tiene como tema principal el impacto ambiental de las botellas de vidrio, el impacto que este tiene actualmente es un tema bastante hablando y muy discutido, según los entendidos el vidrio no contamine al medio ambiente, como también existen otros autores que afirman que el vidrio y los envases de vidrio llegan a ser muy perjudiciales para la naturaleza ya que es un producto que resiste generaciones.

Las botellas de vidrio se las realizan con materia prima a muy altas temperaturas ese es el proceso que lleva la fabricación de las botellas, algunos investigadores dicen que las fabricaciones de estos dañan la atmosfera del planeta porque este produce demasiada energía.

Hoy en día existe muchas personas trabajando para la conservación y cuidado del medio ambiente ellos han optado por el reciclaje de los desechos inorgánicos, tales como tela, metal, cartón o papel, plástico, vidrio, aunque el reciclaje de vidrio es poco a nivel mundial, las grandes industrias prefieren fabricar que reutilizar el envase. El reciclaje de las botellas de vidrio es muy bueno porque si obtenemos 3.000 botellas se ahorra mucho de lo que consume la elaboración de este producto. Con las botellas de vidrio se pueden hacer infinidades de manualidades para el hogar y también para vender.

En Alemania utilizan el canjeo y el descuento en la compra de otro producto de vidrio así se llega al reciclaje de estos productos utilizando un mecanismo como de ingresos y descuento para el hogar en ecuador el reciclaje de vidrio es muy poco llegando a un porcentaje en reciclaje de $12,68 \%$ en el año 2014.

Palabras claves: botellas de vidrio, impacto ambiental y reciclaje.

\footnotetext{
${ }^{1}$ Visionario digital, Ambato,Ecuador,tatianacarrasco@ cienciadigital.org
} 
Abstract.

This document has as its main theme the environmental impact of glass bottles, the impact that this has currently is a subject quite speaking and much discussed, according to experts the glass does not contaminate the environment, as there are other authors who claim that the Glass and glass containers become very damaging to nature as it is a product that withstands generations.

Glass bottles are made with raw materials at very high temperatures that is the process that leads to the manufacture of bottles, some researchers say that the manufacture of these damage the atmosphere of the planet because it produces too much energy.

Today there are many people working for the conservation and care of the environment they have opted for the recycling of inorganic waste, such as cloth, metal, cardboard or paper, plastic, glass, although glass recycling is little worldwide, the large industries prefer to manufacture than to reuse the container. The recycling of glass bottles is very good because if we obtain 3,000 bottles, we save a lot of what the production of this product consumes. With the glass bottles you can make infinities of crafts for the home and also to sell.

In Germany they use the exchange and the discount in the purchase of another glass product so that they get to recycling these products using a mechanism such as income and discount for the home in Ecuador glass recycling is very little, reaching a percentage in recycling of $12.68 \%$ in the year 2014.

Keywords: Impact, technological advances, initial education.

\section{Introducción}

Sauvanet \& Cashmore (2016) afirman: "Para lograr un impacto social con las actividades filantrópicas, las acciones deben demostrar que han contribuido a cambios positivos y sostenibles en beneficio de la sociedad."

Ilustración 1 Impacto Social

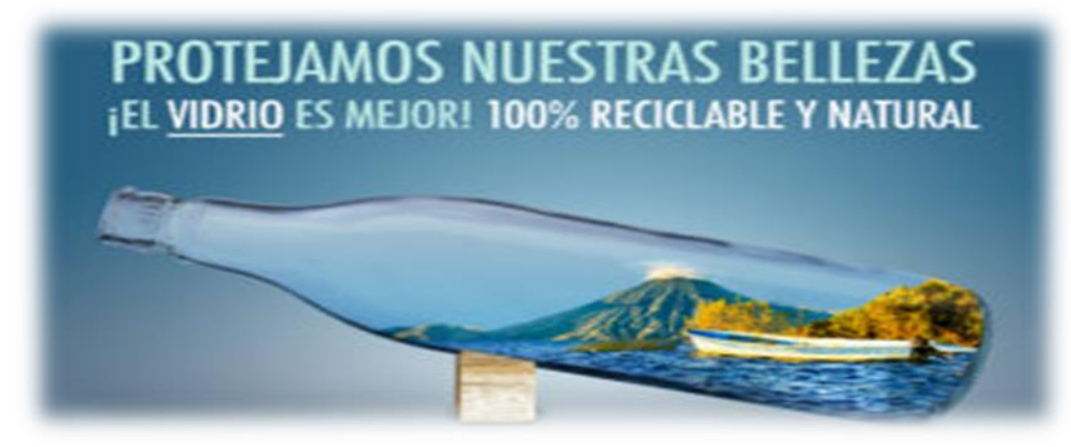

Autor: Velásquez M. (2017).

Fuente: https://distritocentralgt.com/vical-promueve-el-uso-del-vidrio-como-amigo-delambiente/ 
Muchas de las personas hoy en día se dedican a recolectar los desechos inorgánicos y con ellos realizan manualidades, e incluso la venta de las manualidades que se pueden hacer. Se colabora con el medio ambiente y se aporta a la sociedad.

\section{Botellas de Vidrio}

Afirman los autores: (Castro D. 2016., Di Giacomo D. 2018., Gaines K. 2018., Gutiérrez M. 2015., Murphy L. 2017 \& Wiggins P. 2018) Las botellas de vidrio están hechas de materias primas, posee muchas cualidades son adecuado para la conservación de alimentos, su precio varía en el vidrio soplado, las marcas de abejas, el tipo de moldes, las imperfecciones del vidrio, las placas de babosas, las variaciones de labios o partes superiores y la ubicación de la casa de cristal donde se encuentran y son completamente reciclables.

Ilustración 2 Botellas de Vidrio

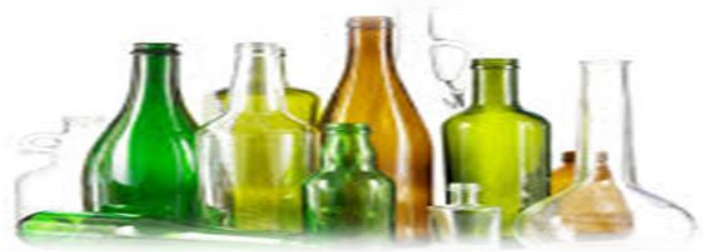

Autor: Varela M. (2015)

Fuente:https://www.google.com.ec/search?rlz=1C1CHZL_esEC773EC773\&biw=1366\& bih=657\&tbm=isch\&sa=1\&ei=_KbPW4e3FcSa_Qbg25KgBw\&q=Botellas+de+Vidrio\& oq=Botellas+de+Vidrio\&gs_l=img.3..016j0i67k1j013.332541.332541.0.333427.1.1.0.0.0. $0.559 .559 .5-$

1.1.0...0...1c.1.64.img..0.1.556...0.dHIXuwVuA8Q\#imgdii=0yRFb9cAgXrBMM:\&imgr $\mathrm{c}=$ SiWfi9KCFV0TcM:

Las botellas de vidrio esta constituidas de materias primas, depende la empresa que las fabrica, realizan los moldeados de la botella, una botella de vidrio puede resistir una generación, el vidrio en si no contamina, pero la botella como es cerrada acoge agua y eso realiza mosquitos.

\section{Impacto Ambiental}

Según los autores: (Äppelqvist M. 2016., Beales J. 2015., Darrouy P. 2014., Martinez Y. 2014., Quintanilla J. 2017 \& Vidal R. 2014) El vidrio no contamina, pero un frasco de vidrio sobrevivirá a generaciones, afecta a la naturaleza. El vidrio genera un gran consumo de energía y emisión de sustancias derivadas de la combustión, este produce contaminantes a la atmósfera. Existe un modo de vida que vela por la sostenibilidad del planeta, reutilizando los materiales que se disponen, se crean nuevos diseños y puestos de trabajo. 
Ilustración 3 Impacto Ambiental del Vidrio

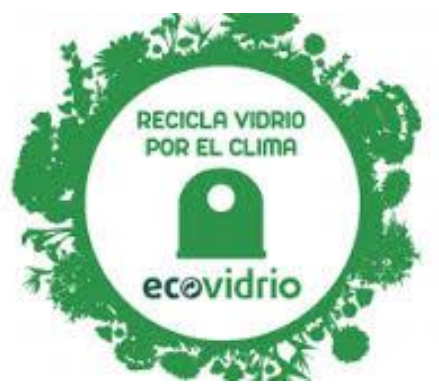

Autor: Varela M. (2018)

Fuente: https://hablandoenvidrio.com/sabes-cual-es-tu-impacto-ecologico/

El impacto ambiental del vidrio según los entendidos no contamina al medio ambiente, pero la elaboración de este si lo hace, las grandes industrias que elaboran las botellas de vidrio consume demasiada energía, y eso llega afectar a la atmósfera del planeta tierra.

\section{Reciclaje}

Según los expertos en reciclaje: (Drake J. 2017., Figueroa A. 2017., Jennings S. 2016., Li J. 2016., Ruiz R. 2017 \& Sánchez J. 2015) El reciclaje es una forma muy popular de salvar al planeta de la contaminación, reciclando 3.000 botellas de vidrio se ahorra más de una tonelada de materia prima, el vidrio se puede reciclar por completo sin perder el material, calidad. Tratados con cuidado, sus envases de vidrio vivirán indefinidamente. El reciclaje del vidrio permite convertir los residuos de vidrio en productos que pueden ser incorporados a la sociedad para su uso posterior, ya sea mediante un proceso de lavado e higienizado para su posterior reutilización o volviendo a fundir el material para la elaboración del mismo producto o de productos similares.

Ilustración 4 Reciclaje de Botellas de Vidrio

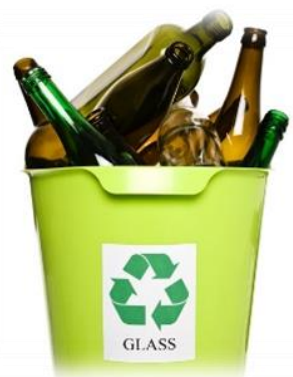

Autor: Benavente C. (2015)

Fuente: https://noticiasgreenpress.com/2015/11/01/el-2-de-noviembre-inicia-la-campanade-reciclaje-de-vidrio/ 
El reciclaje es un tema muy hablado en la actualidad las personas están concientizadas del mal que se le hace al planeta con los desechos inorgánicos, por eso hoy en día la gente recicla o crea nuevas cosas con ese desecho para que sea útil dentro del hogar.

\section{Estadísticas}

La composición de la basura es la prueba que pasamos de un estilo de vida en donde predominaban los productos orgánicos a uno de productos industrializados. Países que más reciclan de la Organización para la Cooperación y el Desarrollo Económico (OCDE). (Guijarro, 2016)

Ilustración 5 Países de la OECD que más reciclan

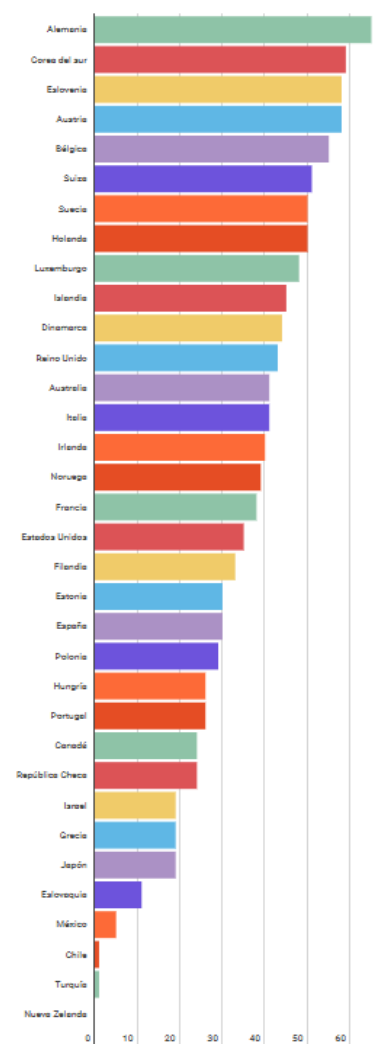

Autor: Guijarro L. (2016)

Fuente: https://infogram.com/untitled-1g18m3j5r687p36

Se puede observar en la ilustración que el País de Alemania es aquel que más recicla y se esmera con el canjeo de sus productos de vidrio a estos allá lo llaman Pfand un método de no desechar el vidrio más bien que la persona salga beneficiada por su nueva compra, otro de los países es Corea del Sur. 
A nivel nacional, entre los años 2010 y 2014, se incrementó el porcentaje de hogares que clasificaron residuos inorgánicos. El uso de materiales reciclables como materia prima en la manufactura de nuevos productos ayuda a conservar recursos naturales renovables y no renovables. (Arias \& Seilles, 2014)

Ilustración 6 Hogares que clasificaron los Residuos Inorgánicos a nivel nacional $(\%) 2$

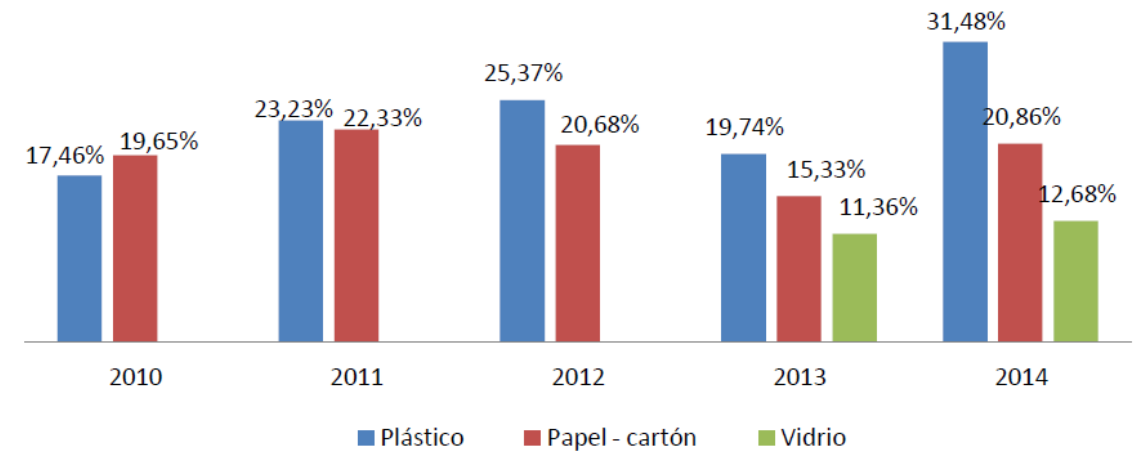

Autor: Arias P. \& Seilles M. (2014)

Fuente: El Instituto Nacional de Estadística y Censos, mediante la Dirección de Estadísticas Agropecuarias y Ambientales. Clasificación de Residuos Inorgánicos.

Ilustración 7 Disposición final de los residuos inorgánicos clasificados en 2014 $(\%)$

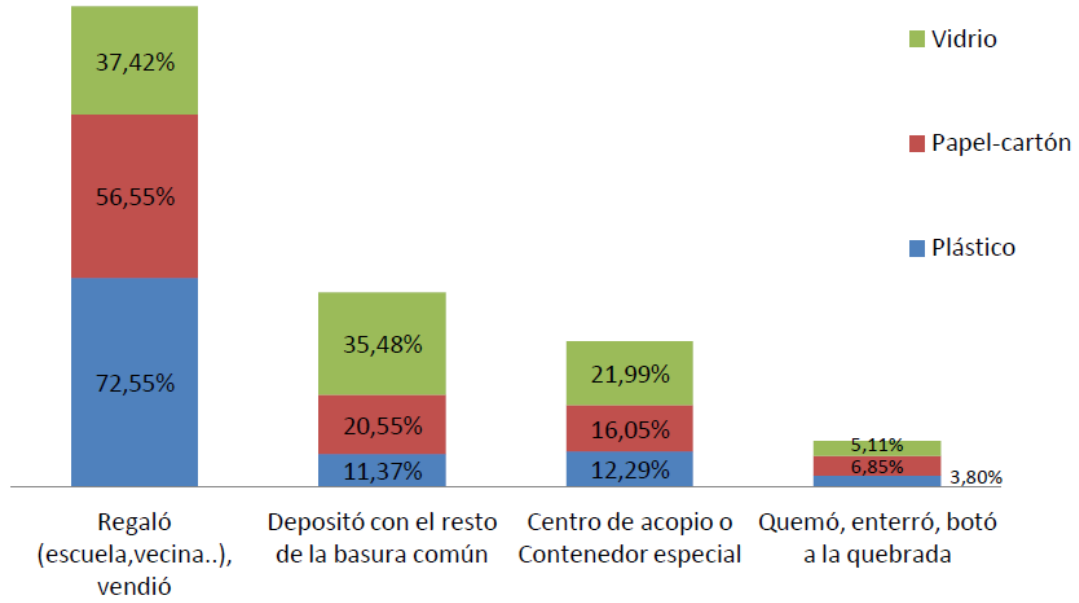

Autor: Arias P. \& Seilles M. (2014)

Fuente: El Instituto Nacional de Estadística y Censos, mediante la Dirección de Estadísticas Agropecuarias y Ambientales. Clasificación de Residuos Inorgánicos. 
El vidrio a nivel nacional no es muy reciclado, se puede visualizar en las estadísticas que en año 2013 y 2014 recién incrementaron el reciclaje de este desecho inorgánico, la gente aquí lo hace por ganar dinero, pero incluso el pago de estos es muy bajo, Ecuador no se puede comprar al reciclaje que se realiza en el País de Alemania.

\section{Conclusiones}

Las elaboraciones de las botellas de vidrio dañan la atmosfera al planeta tierra, industrias grandes que consumen demasiada energía.

$>$ El vidrio no contamina al medio ambiente, pero los envases de vidrio perjudican a la naturaleza he incluso a los animales, porque este producto resiste generaciones.

$>$ Los elementos inorgánicos reciclándolos y reutilizándolos son muy útiles en el hogar he incluso para emprender un trabajo.

$>$ Ecuador tiene un 12,68\% en reciclaje de vidrio en el 2014, Alemania es un país que ahora se dedica a reciclar mediante maquinas con sensores.

\section{Referencias Bibliográficas}

Äppelqvist, M. (14 de Diciembre de 2016). Glastory. Obtenido de ¿Cómo afectan los factores ambientales al proceso de templado del vidrio?: https://www.glastory.net/environment-influences-glass-tempering-process/

Arias, P., \& Seilles, M. (2014). Información Ambiental en hogares 2014. Ecuador: DIRECCIÓN DE COMUNICACIÓN SOCIAL.

Beales, J. (9 de Octubre de 2015). Por qué siempre debe reciclar vidrio (incluso cuando no es conveniente). Selva Beat. Obtenido de https://www.selvabeat.com/home/2015/9/10/why-you-should-always-recycleglass

Benavente, C. ( 01 de 11 de 2015). El 2 de noviembre inicia la campaña... Obtenido de El 2 de noviembre inicia la campaña de reciclaje de vidrio: https://noticiasgreenpress.com/2015/11/01/el-2-de-noviembre-inicia-lacampana-de-reciclaje-de-vidrio/

Castro, D. (2 de abril de 2016). Cómo hacer con una botella de vidrio una original tabla para snacks ... Obtenido de Cómo hacer con una botella de vidrio una original tabla para snacks: https://mejorconsalud.com/una-botella-vidrio-una-originaltabla-snacks/

Darrouy, P. (14 de Mayo de 2014). El impacto ambiental de la elaboración de botellas de vidrio by ... - Prezi. Obtenido de El impacto ambiental de la elaboración de 
botellas de vidrio: https://prezi.com/yt56-sygz0wz/el-impacto-ambiental-de-laelaboracion-de-botellas-de-vidrio/

Di Giacomo, D. ( 02 de Abril de 2018). ¿Por qué las botellas de vino son de vidrio? - De Vinos y Vides. Obtenido de ¿Por qué las botellas de vino son de vidrio?: https://www.devinosyvides.com.ar/nota/799-por-que-las-botellas-de-vino-sonde-vidrio

Drake, J. (19 de Abril de 2017). Efectos del vidrio sobre el medio ambiente. Anima Bets.

Figueroa , A. (2017). "El reciclaje del vidrio como elemento de emprendimiento de bisutería femenina”. UNIVERSIDAD TÉCNICA DE AMBATO FACULTAD DE CIENCIAS ADMINISTRATIVAS, Ambato. Obtenido de http://repositorio.uta.edu.ec/bitstream/123456789/26960/1/430\%20o.e..pdf

Gaines, K. (01 de February de 2018). $\rightarrow$ Cómo hacer botellas de vidrio desde cero o vidrio reciclado ... Obtenido de Cómo hacer botellas de vidrio desde cero o vidrio reciclado: https://www.geniolandia.com/13072984/como-hacer-botellas-devidrio-desde-cero-o-vidrio-reciclado

Guijarro, L. (22 de 06 de 2016). Los países que más basura generan y los que más reciclan del mundo. Obtenido de Los países que más basura generan y los que más reciclan del mundo: https://www.huffingtonpost.es/2016/06/22/paises-contaminanrecicla_n_10509726.html

Gutiérrez, M. (2015). CRISTALERÍA ECOLÓGICA A BASE DE BOTELLAS DE VIDRIO RECICLADAS. UNIVERSIDAD CENTRAL DEL ECUADOR. QUITO: Quito: UCE. Obtenido de http://www.dspace.uce.edu.ec/bitstream/25000/5444/1/T-UCE-0011-24.pdf

Jennings, S. (7 de Octubre de 2016). Little green lives. Obtenido de Tarros de masón: los pequeños tarros de vidrio que causan un gran impacto ambiental.: https://www.littlegreenlives.com/living-simply-2/index.php/2016/10/08/masonjars

Li, J., Xian, Z., Ying, L., Zhi, H., Feng, G., Yu, L., \& Xiao, Q. ( 2016). Análisis del impacto ambiental del vidrio de espuma. Obtenido de Materiales y Tecnologías para el Suministro de Energía e Ingeniería Ambiental.: https://www.scientific.net/MSF.847.315

Martinez, Y. (11 de Septiembre de 2014). EL VIDRIO Y EL MEDIO AMBIENTE by YESENIA MTZ. on Prezi. Obtenido de EL VIDRIO Y EL MEDIO AMBIENTE: https://prezi.com/u88g-hd66ymb/el-vidrio-y-el-medio-ambiente/

Murphy, L. (11 de Agosto de 2017). Enfrentamiento de contenedores de bebidas: Plástico vs. Vidrio vs. Aluminio ... Obtenido de Enfrentamiento de contenedores de 
bebidas: Plástico vs. Vidrio vs. Aluminio: https://earth911.com/living-wellbeing/recycled-beverage-containers/

Quintanilla, J. (9 de Mayo de 2017). Efectos positivos del reciclaje y su impacto en el calentamiento global ... Obtenido de efectos-positivos-del-reciclaje-impactocalentamiento-global: https://www.medicinalliure.com/es/efectos-positivos-delreciclaje-impacto-calentamiento-global/

Ruiz, R. (14 de 09 de 2017). Diez preguntas impertinentes sobre el reciclaje de vidrio. Obtenido de Diez preguntas impertinentes sobre el reciclaje de vidrio, ¿tienen respuesta?: https://elasombrario.com/diez-preguntas-impertinentes-reciclajevidrio/

Sánchez, J. (09 de Septiembre de 2015). EL RECICLAJE DE LOS ENVASES DE VIDRIO EN CUBA. Caribeña de Ciencias Sociales. Obtenido de http://www.eumed.net/rev/caribe/2015/09/vidrio.html

Sauvanet, N., \& Cashmore, A. (2016). ¿QUÉ ES EL IMPACTO SOCIAL Y POR QUÉ ES IMPORTANTE? BNP Paribas Wealth Management, https://wealthmanagement.bnpparibas/es/es/expert-voices/social-impact.html.

Varela, M. (17 de Marzo de 2015). Prevención y ecodiseño:. Obtenido de Prevención y ecodiseño: el mejor residuo, el que no se produce: https://hablandoenvidrio.com/prevencion-y-ecodiseno-el-mejor-residuo-el-queno-se-produce/

Varela, M. (22 de Marzo de 2018). Sabes cuál es tu impacto ecológico?... Obtenido de ¿Sabes cuál es tu impacto ecológico?: https://hablandoenvidrio.com/sabes-cuales-tu-impacto-ecologico/

Velásquez, M. (5 de septiembre de 2017). VICAL promueve el uso del vidrio. Obtenido de VICAL PROMUEVE EL USO DEL VIDRIO COMO AMIGO DEL AMBIENTE: https://distritocentralgt.com/vical-promueve-el-uso-del-vidriocomo-amigo-del-ambiente/

Vidal, R. (04 de Enero de 2014). Aspectos ambientales del Vidrio y la Cerámica | ecoWigid. ecoWigid. Obtenido de Aspectos ambientales del vidrio y la cerámica: http://www.gid.uji.es/ecowigid/?q=node/648

Wiggins, P. (15 de 02 de 2018). Aprendiendo a valorar las viejas botellas de vidrio - The Spruce Crafts. Obtenido de Aprende a valorar las botellas de vidrio viejas: https://www.thesprucecrafts.com/learn-to-value-old-bottles-149103 


\section{Para citar el artículo indexado.}

Carrasco T. (2017). El reciclaje de vidrio y su impacto en la conservación del medio ambiente. Revista electrónica Explorador Digital 1(2), 22-31. Recuperado desde:

http://cienciadigital.org/revistacienciadigital2/index.php/exploradordigital/article/view/319/7 $\underline{31}$

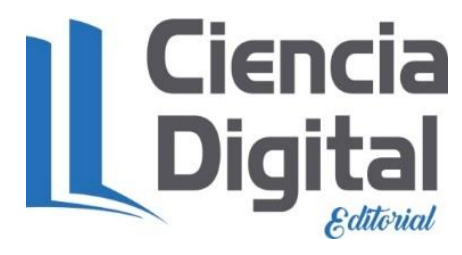

El artículo que se publica es de exclusiva responsabilidad de los autores y no necesariamente reflejan el pensamiento de la Revista Explorador Digital.

El articulo queda en propiedad de la revista y, por tanto, su publicación parcial y/o total en otro medio tiene que ser autorizado por el director o editor de la Revista Explorador Digital.
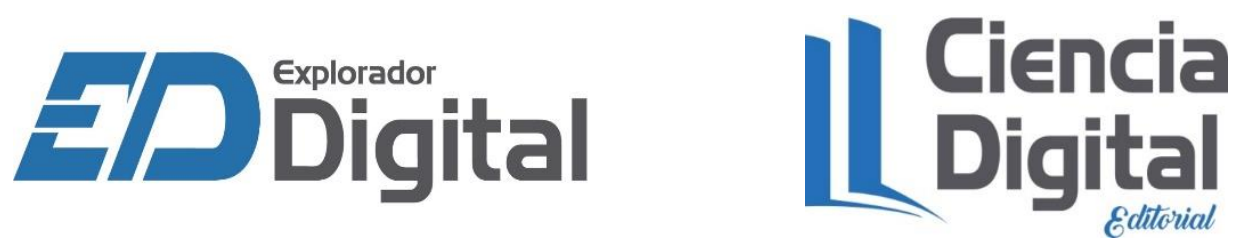